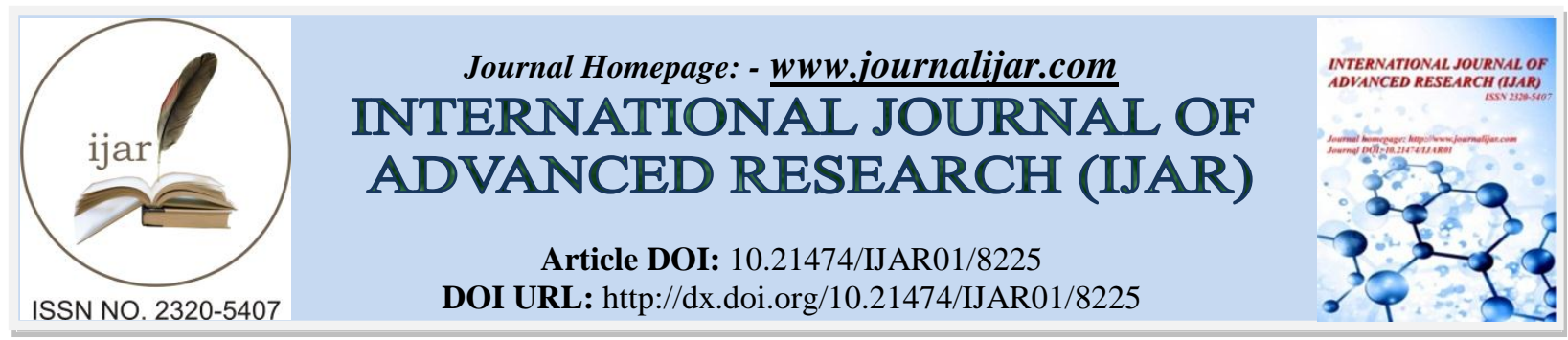

RESEARCH ARTICLE

\title{
HEMORRAGIE VITREENNE MASSIVE AVEC DECOLLEMENT CHOROIDIENNE REVELANT UN MYELOME MULTIPLE SYMPTOMATIQUE.
}

\begin{abstract}
A.bouzidi , a. Elouafi , a.laayoune, s.iferkhasse and a.laktaoui.
Service d'ophtalmologie. Hôpital Militaire Moulay Ismail Meknès, 2019 Mots Clés : Myélome multiple, complications, œil.
\end{abstract}

\section{Manuscript Info}

Manuscript History

Received: 18 October 2018

Final Accepted: 20 November 2018

Published: December 2018

\section{Abstract}

Copy Right, IJAR, 2018,. All rights reserved.

\section{Introduction:-}

Le myélome multiple (MM) est une maladie néoplasique rare d'étiologie inconnue caractérisée par une prolifération plasmocytaire siégeant classiquement dans la mœlle osseuse et induisant une surproduction d'immunoglobulines monoclonales [ 1 ]. Ces complications sont multiples : osseuses, neurologiques; rénales, les infections et syndrome d'hyperviscosite.

\section{Cas clinique}

Un patient de 54 ans s'est présente en consultation d'ophtalmologie pour une baisse d'acuité visuelle de l'œil droit avec rougeur et larmoiement le tout évoluant dans contexte d'altération de l'état général. (Fièvre, dyspnée, anémie).

Un bilan général a été réalisé

1. NFS :

2. $\mathrm{Hb}: 5.9 \mathrm{~g} \quad \mathrm{VGM}=110$

3. PLQ : $75000 / \mathrm{mm} 3$

4. GB : $6000 / \mathrm{mm} 3$ PNN : 2440

5. Myélogramme : $80 \%$ de plasmocytes dystrophique.

6. Protidogramme : Gamma glob : $64 \mathrm{~g} / \mathrm{l} \quad \mathrm{Alb}: 18.8 \mathrm{~g} / \mathrm{l}$

7. Immuunofixation : Ig G Lambda

8. Analyse cytogénétique : pas de Del 17p, t(4,14) ou Del1p.

9. Protéinurie : en cours

10. Dosages des Chaines légères libres sériques : non fait

11. Calcémie corrigée : $113 \mathrm{mg} / \mathrm{l}$

12. Créatinémie : $16.5 \mathrm{mg} / \mathrm{l}$

13. Béta2 $\mu$ globulinémie : non faite Rx standards du crane : lésions à l'emporte pièce + déminéralisation diffuse (. Fig : 1)

14. IRM rachis + orbito cérébrale :

15. Aspect hyper signal T1/T2et FLAIR hétérogène du globe oculaire droit évoquant une hémorragie vitréenne. ( Fig : 2)

16. Décollement rétinien avec épaississement choroïdien

17. Nerfs optique est sans anomalie

Corresponding Author:-A.bouzidi.

Address:-Service d'ophtalmologie. Hôpital Militaire Moulay Ismail Meknès, 2019 Mots Clés :

Myélome multiple, complications, œil. 
18. Déminéralisation du rachis surtout cervical

19. L'examen ophtalmologique montre :

Une acuité visuelle nulle de l'œil droit avec au FO : une hémorragie vitrienne dense totale stade 4 avec décollement rétinien et choroïdien confirme par l'échographie oculaire. Le patient a finalement été diagnostiqué comme souffrant de myélome multiple symptomatique et adresse au service d'hématologie clinique pour prise en charge.

\section{Discussion:-}

\section{Mécanismes d'atteinte oculaire}

1. Le cancer peut affecter l'œil et l'orbite par une infiltration néoplasique métastasique directe ou par compression ou par une circulation d'anticorps entraînant une dégénérescence rétinienne paranéoplasique [ 2,4$]$.

2. L'un des mécanismes par lesquels le MM affecte l'orbite et les tissus oculaires est par infiltration directe ou sous forme de plasmocytomes extra médullaires.

3. Le résultat de lésion de masse peut provoquer le déplacement ou la compression des tissus.

4. Un autre mécanisme est le syndrome d'hyperviscosité .ceci par une hyper gama globuline, l'augmentation de la teneur en protéines et de la grande taille moléculaire, une polymérisation anormale et la forme anormale des molécules d'immunoglobuline[ 2 ].

5. Il survient chez 2 à $6 \%$ des patients atteints de MM. Responsable des altérations du fond de l'œil (55,6\%), Manifestations neurologiques $(88,9 \%)$, tendance aux saignements $(44,4 \%)$ et insuffisance rénale $(55,6 \%)$ [ 2 ].

\section{Les manifestations oculaires de MM}

\section{La rétine}

1. Dans un examen approfondi des anomalies rétiniennes associées aux maladies du sang, Holt et al124 ont signalé la présence de rétinopathie chez 8 des 22 patients atteints de myélome. Le plus souvent, il s'agissait d'hémorragies discrètes. Des hémorragies centrées en forme de flamme et blanches et des infarctus de la couche de fibres nerveuses ont parfois été observés[ 1 ].

2. La présence de rétinopathie n'était pas associée à un pire pronostic. Carr et Henkind "ont rapporté que les microanévrysmes de la rétine périphérique étaient fréquents dans les cas de myélome multiple et d'autres troubles hypergammaglobulinémiques. Sanders et al40 ont utilisé des digestions rétiniennes à la trypsine pour découvrir des microanévrysmes capillaires de la rétine médio-périphérique chez 7 des 11 patients atteints de myélome multiple [ 1,2$]$.

3. On observe également une dilatation et une tortuosité des veines rétiniennes, des hémorragies superficielles et profondes de la rétine et des résidus d'œdème. Des cas d'occlusion de la veine rétinienne centrale, d'occlusion rétinienne de branche, de néovascularisation et d'hémorragies sous-hyaloïdiennes et du corps vitré ont été rapportés en association avec hyperviscosité sérique. Des décollements séreux et exsudatifs de la rétine associés au myélome multiple ont également été rapportés.

4. Dans notre cas ce patient a subi un décollement choroïdo rétinienne avec une hémorragie massive du corps de vitre évoluant vers la phtyse du globe qui est reste rare. (Fig 3)

\section{Cornée et conjonctive}

L'implication de la cornée serait rare en association avec le myélome multiple. Plusieurs variétés ont été décrites. Aronson et al * et Pinkerton et al134 ont observé des dépôts cristallins linéaires dans le stroma de la cornée. Pinkerton et al. Ont trouvé un matériau cristallin dans des cellules de type plasmatique dans le tissu conjonctif conjonctival, avec des cellules très rares dans le stroma de la cornée [ 3 ].

Eiferman et al ${ }^{\prime} *$ ont récemment décrit des dépôts dans la membrane de Bowman et un stroma superficiel chez un patient atteint de gammapathie monoclonale d'IgG-K. Les dépôts se sont révélés être des chaînes IgG-K par immunofluorescence directe. Les patients rapportés par Aronson et al. Et Eiferman et al. Se sont plaints de photophobie et d'irritation. La vision n'était pas significativement affectée.

\section{Uvée}

1. Bronstein a décrit l'implication de l'uvée dans le myélome multiple. Il a découvert des agrégats de plasmocytes dans l'iris et la choroïde. Des plasmocytes ont été vus flottant librement dans la chambre antérieure et adhérente à la cornée postérieure [ 1 ].

2. Nous avons récemment découvert des microcystes bilatéraux de l'épithélium pigmenté à l'iris chez 6 des 11 patients atteints de myélome étudiés histologiquement à l'hôpital Montefiore. La tranche d'âge des patients affectés était de 47 à 75 ans. Aucune corrélation avec l'âge ou le sexe n'a été trouvée. 


\section{Conclusion:-}

Les manifestations ophtalmiques du MM sont rares et diverses. Ils peuvent apparaître lors de la présentation initiale de la maladie ou se manifester tardivement. La prise de conscience des manifestations possibles peut conduire à un diagnostic plus précoce et avoir une influence positive sur l'évolution de la maladie.

\section{Iconographie}

Figure 1:-Radio du crâne montrant des zones de déminéralisations osseuses à l'emporte pièce

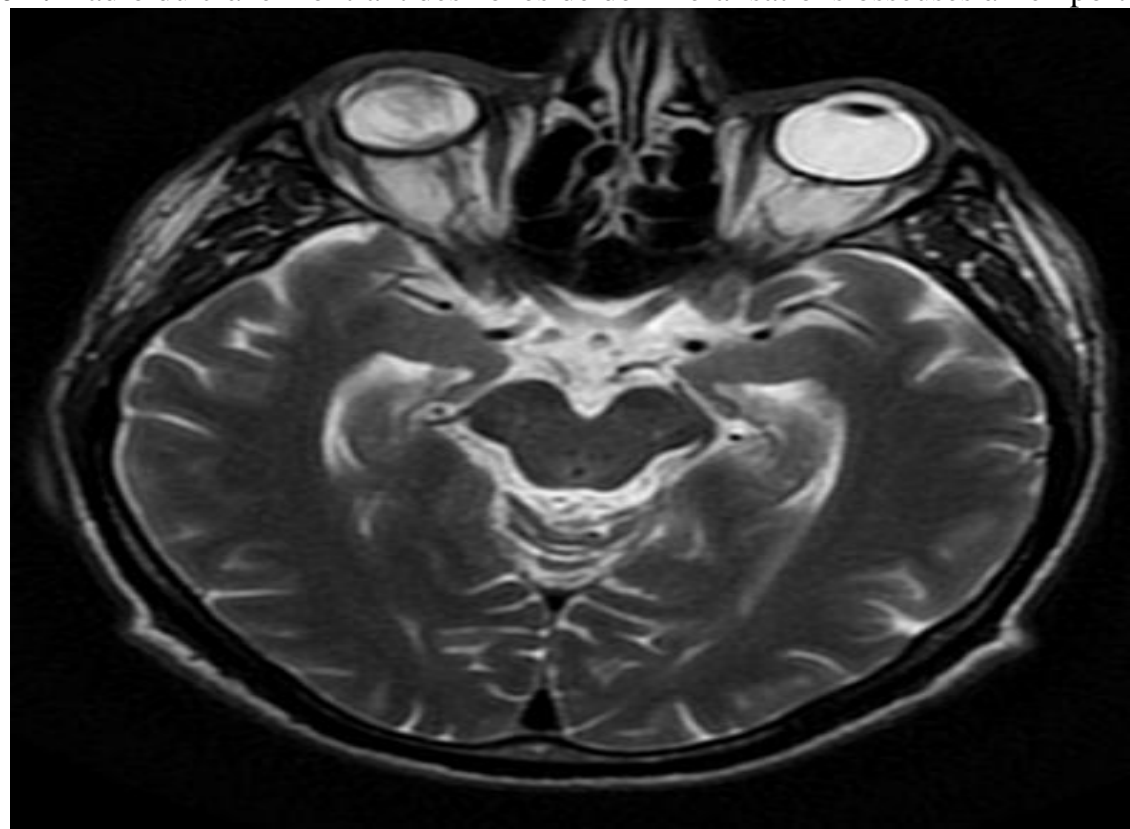

Figure 2:-IRM orbito cérébral montrant une vitre charge trouble avec épaississement choroïdien et décollement de rétine de l'œil droit

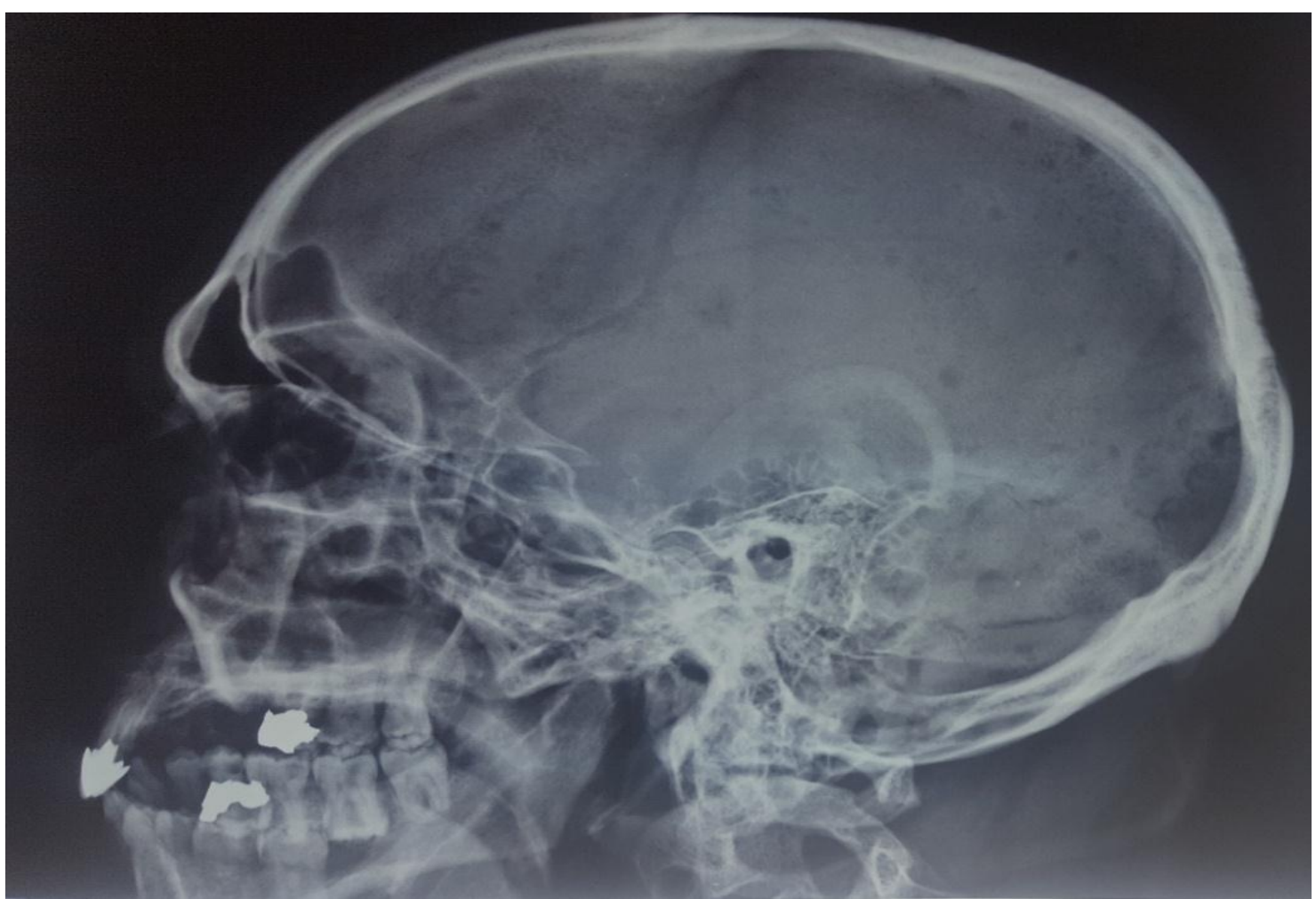


Figure 3:-phtyse du globe oculaire de l'œil droit. Stade ultime de l'évolution de cette complication

\section{Références :-}

1. Alan J. Knapp, M.D., Samuel Gartner, M.D., AND Paul Henkind, M.D., PH.D. Multiple Myeloma and Its Ocular Manifestations. Survey of Ophthalmolology, Vol 31 No 6, 1987.

2. Omti A. E., Omti. C.E. Ophtalmic Manifestations of Multiple Myeloma. West Africain Journal of Medecine, Vol 26 No 4, 2007.

3. Naiyang Li., Zhe Zhu., Gengrong Yi., Sheng Li., Xiaotong Han. Corneal Opacity Leading to Multiple Myeloma Diagnosis: A Case Report and Literature Review. Am J Case Rep, Vol 19, No 21, 2018.

4. Ngolet Lo., Kocko I., Kaliba Atipo Fo., Guelongo Okouango Ova Jd., Ntsiba H, Elira Dokekias A. Le myelome multiple symptomatique a brazzaville : à propos de 40 cas. Annales de l’Université Marien NGOUABI, Vol 16 (1), No 7, 2016. 